\title{
Improvements in the Data Partitioning Approach for Frequent Itemsets Mining
}

\author{
Son N. Nguyen and Maria E. Orlowska \\ School of Information Technology and Electrical Engineering, \\ The University of Queensland, QLD 4072, Australia \\ \{nnson, maria) ditee.uq.edu.au
}

\begin{abstract}
Frequent Itemsets mining is well explored for various data types, and its computational complexity is well understood. There are methods to deal effectively with computational problems. This paper shows another approach to further performance enhancements of frequent items sets computation.

We have made a series of observations that led us to inventing data preprocessing methods such that the final step of the Partition algorithm, where a combination of all local candidate sets must be processed, is executed on substantially smaller input data. The paper shows results from several experiments that confirmed our general and formally presented observations.
\end{abstract}

Keywords: Association rules, Frequent itemset, Partition, Performance.

\section{Introduction}

Since the association rules mining introduction by Argawal et al. [5], many algorithms and their subsequent improvements have been proposed to solve association rules mining, especially frequent itemsets mining problems.

In this paper, we review the state of the art in association rules mining with a focus on frequent itemsets mining. There are many well-accepted approaches such as "Apriori" by Argawal et al. [1], ECLAT by Zaki [7], and more recently "FP-growth" by Han et al. [8]. Another interesting class of solutions is based on the data partitioning approach. This fundamental concept was originally proposed as a Partition algorithm by Savaserse et al. [2], and it was improved later in AS-CPA by Lin et al. [4] and ARMOR by Pudi et al. [11]. A common feature of these results is their target, namely the limitation of I/O operations by considering data subsets dictated by the main memory size.

An intriguing question is whether we could improve the overall performance of mining large data sets by a smarter but not too 'expensive' design of the data fragments - rather than determine them by a sequential transaction allocation based on the fragment size only.

The main goal of this paper is to demonstrate our observations, generalize, and specify corresponding data pre-processing for the Partitioning approach in order to improve the performance. Our study is supported by a series of experiments which indicate a dramatic improvement in the performance of the Partitioning approach with our fragmentation method, in contrast to the traditional one [2]. 
The remainder of the paper is organised as follows. Section 2 introduces the basic concepts related to frequent itemsets mining. Section 3 reviews the current state of art in the field, especially for frequent itemsets mining and the Partitioning approach. Section 4 presents our observations and open issues. We propose the pre-processing data fragmentation solution in section 5. Section 6 shows the result from our experiment, and finally, we present our concluding remarks in section 7.

\section{Preliminary Concepts}

For the completeness of this presentation and to establish our notation, this section gives a formal description of the problem of mining frequent itemsets . It can be stated as follows [1]:

Let $I=\left\{i_{1}, i_{2}, \ldots, i_{m}\right\}$ be a set of $m$ distinct literals called items. Transaction database $\mathrm{D}$ is a set of variable length transactions over $\mathrm{I}$.

Each transaction contains a set of items $\left\{\mathrm{i}_{\mathrm{j}}, \mathrm{i}_{\mathrm{k}}, \ldots, \mathrm{i}_{\mathrm{h}}\right\} \subseteq \mathrm{I}, \mathrm{i}_{\mathrm{j}}<\mathrm{i}_{\mathrm{k}}<\ldots<\mathrm{i}_{\mathrm{h}}$. Each transaction has an associated unique identifier called TID.

For an itemset $\mathrm{X} \subseteq \mathrm{I}$, the support is denoted $\sup _{\mathrm{D}}(\mathrm{X})$, equals to the fraction of transactions in D containing $\mathrm{X}$.

The problem of mining frequent itemsets is to generate all frequent itemsets $\mathrm{X}$ that have $\sup _{\mathrm{D}}(\mathrm{X})$ no less than user specified minimum support threshold.

\section{Review Frequent Itemsets Mining}

Throughout the last decade, there have been many attempts and well-known algorithms that target an efficient solution of the frequent itemsets mining problem. However, the performance of these algorithms depends on many, often very specific input data features and additionally, implementation environments. As a result, several claims made in earlier papers were later debated by other authors.

\subsection{Partitioning Approach for Frequent Itemsets Mining}

Savaserse et al. [2] proposed the Partition algorithm based on the following principle. A fragment $\mathrm{P} \subseteq \mathrm{D}$ of the database is defined as any subset of the transactions contained in the database D. Further, any two different fragments are non-overlapping. Local support for an itemset is the fraction of transactions containing that itemset in a fragment. Local candidate itemset is being tested for minimum support within a given fragment. A Local frequent itemset is an itemset whose local support in the fragment is no less than the minimum support. Global support, Global candidate itemset, Global frequent itemset are defined as above except they are in the context of the entire database. The goal is to find all Global frequent itemsets.

The following Lemma 1 supports the main principle of the Partition algorithm.

Lemma 1: If $\mathrm{X}$ is a frequent itemset in database $\mathrm{D}$, which is partitioned into $\mathrm{n}$ fragments $\mathrm{P}_{1}, \mathrm{P}_{2}, \ldots, \mathrm{P}_{\mathrm{n}}$, then $\mathrm{X}$ must be a frequent itemset in at least one of the $\mathrm{n}$ fragments.

Proof: Due to the limit space, the proof can be seen in [10] 
The Partition algorithm divides $\mathrm{D}$ into $\mathrm{n}$ fragments. The algorithm first scans fragment $\mathrm{P}_{\mathrm{i}}$ in the main memory at a time, for $\mathrm{i}=1, \ldots, \mathrm{n}$, to find the set of all Local frequent itemsets in $\mathrm{P}_{\mathrm{i}}$, denoted as $\mathrm{LP}_{\mathrm{i}}$. Then, by taking the union of $\mathrm{LP}_{\mathrm{i}}$, a set of candidate itemsets over $\mathrm{D}$ is constructed, denoted as $\mathrm{C}^{\mathrm{G}}$. Based on Lemma $1, \mathrm{C}^{\mathrm{G}}$ is a superset of the set of all Global frequent itemsets in D. Finally, the algorithm scans each fragment for the second time to calculate the support of each itemset in $\mathrm{C}^{\mathrm{G}}$ and to find the Global frequent itemsets.

\subsection{Related Work in Partitioning Approach}

One of the Partition algorithm derivatives is AS-CPA (Anti-Skew Counting Partition Algorithm) by Lin et al. [4]. Recently, there has been another development based on the partitioning approach in the ARMOR algorithm by Pudi et al. [11].

All the above algorithms mainly attempt to reduce the number of false candidates as early as possible. However, they do not consider any features and characteristics of data sets in order to partition the original data set more suitably for further processing.

Further in this paper, we demonstrate that looking more closely into the data itself may deliver good gains in overall performance. As a result, the Local frequent itemsets can be dramatically reduced. Furthermore, in many cases that leads to a larger number of common Global candidates among fragments. Finally, as a consequence, this approach reduces substantially the Global candidates $\left(\mathrm{C}^{\mathrm{G}}\right.$ set $)$.

\section{Observations in the Partitioning Approach}

We begin by considering the first and very obvious measurable data-partitioning attribute - the size of fragments and their impact on the efficiency of the frequent items search process. Further on we examine more closely the composition of fragments at the design time to ensure that selection of transactions satisfy some desired properties.

\subsection{Reasoning About Size of Fragment}

It is not hard to observe that the size of the fragments is inverse-proportional to the size of the output of Local computation. Hence, the question is: What is a 'good' fragment size? We consider several heuristic methods to identify the suitable size of fragments.

We note the following observation: the smaller fragment generates a more negative effect on the number of Local frequent itemsets. Clearly, the best partitioning of data set $\mathrm{D}$ into $\mathrm{n}$ fragments is defined as a method that generates the smallest number of Global candidates. We denote this smallest number as $\mathrm{G}_{\mathrm{n}}$. Note that the perfect solution would have to exhibit the following property; every fragment of the data generates identical Local frequent itemsets.

We generalise these observations as follows;

Lemma 2: If database $\mathrm{D}$ is partitioned into $(\mathrm{n}+1)$ fragments $\mathrm{P}_{1}, \mathrm{P}_{2}, \ldots, \mathrm{P}_{\mathrm{n}+1}$ then the number of Global candidates, denoted $I C^{G}{ }_{n+1}$, is always greater than or equal to $G_{n}$; $\left|C^{\mathrm{G}+1}{ }\right| \geq \mathrm{G}_{\mathrm{n}}$

Proof: Due to the limit space, the proof can be seen in [10] 
As a consequence, the size of a fragment should maintain proper balance in order to control the number of Local frequent itemsets.

\subsection{Some Characteristics of Fragment Data}

Data skew has a negative impact on the Partitioning approach. Basically, data skew causes the Local frequent itemsets generated from different fragments to have very few common elements. In such situations, the number of Global candidates (being the union of all $\mathrm{LP}_{\mathrm{i}}$ ) is rather large.

Obviously, fragments that have many dissimilar transactions (transactions with small or empty intersections) generate a small number of Local frequent itemsets. In this paper we call them dissimilar fragments.

These observations confirm our initial hypothesis that there are some relationships between the composition of fragments and the amount of computation required at the end. We illustrate the fact that a larger number of fragments increase the size of the computation space. In addition, for given number of fragments $n$, a different partition also impacts on the number of Global candidates. Furthermore, the gap in performance is increased dramatically when the support threshold is decreased and the number of fragments is increased.

\section{Data Set Pre-processing}

We present the following algorithms for original data pre-processing.

\subsection{Naive Algorithm}

One of the simplest techniques to be considered is the skipping technique. Before formalising this concept we show a simple example to illustrate its main principle.

Consider data set $\mathrm{D}$ represented by a straight line on figures below. We partition D into 2 fragments as illustrated on the Figure 1. When a Skipping technique is used then $\mathrm{D}$ is initially divided into 4 small sequential parts. Each fragment is created by taking the union of 2 small skipping parts as it is shown on Figure 2.

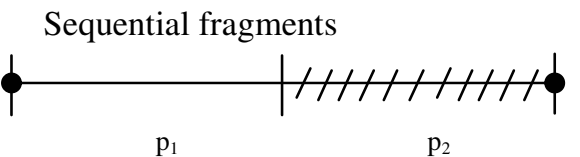

Fig. 1.

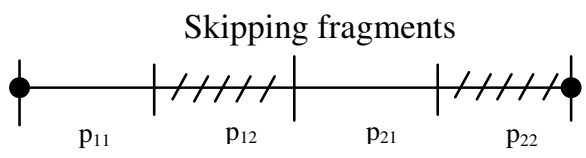

Fig. 2.

One can easily generalise such partition process for any higher number of expected fragments.

\subsection{An incremental Clustering Algorithm}

The incremental clustering algorithm is our idea for pre-processing. The data set will be scanned only once and all clusters (fragments) containing mostly dissimilar transactions are generated at the end of that scan. We introduce some basic definitions. 
Definition 5.1: Cluster Centroid is a set of all Items in the cluster, we denote it $\mathrm{C}_{\mathrm{i}}=$ $\left\{I_{1}, I_{2}, . ., I_{n}\right\}$. Additionally, each item in $C_{i}$ has its associated weight which is its number of occurrences in the cluster; $\left\{\mathrm{w}_{1}, \mathrm{w}_{2}, \ldots, \mathrm{w}_{\mathrm{n}}\right\}$

Definition 5.2: Similarity function between two item sets, in particular a transaction and a cluster centroid, is denoted $\operatorname{Sim}\left(\mathrm{T}_{\mathrm{i}}, \mathrm{C}_{\mathrm{j}}\right)$ and defined as follows;

$\operatorname{Sim}\left(\mathrm{T}_{\mathrm{i}}, \mathrm{C}_{\mathrm{j}}\right) \rightarrow \mathrm{R}^{+}$; Calculation of this function:

1. Let $\mathrm{S}$ be the intersection between the arguments of $\operatorname{Sim}$ function, $S=T_{i} \cap C_{j}$

2. If $\mathrm{S}=\mathscr{O}$ then $\operatorname{Sim}\left(\mathrm{T}_{\mathrm{i}}, \mathrm{C}_{\mathrm{j}}\right)=0$. Otherwise, $\mathrm{S}=\left\{\mathrm{I}_{1}, \mathrm{I}_{2}, \ldots, \mathrm{I}_{\mathrm{m}}\right\}$ with the corresponding weights $\left\{\mathrm{w}_{1}, \mathrm{w}_{2}, \ldots, \mathrm{w}_{\mathrm{m}}\right\}$ in cluster $\mathrm{C}_{\mathrm{j}}$, respectively, therefore $\operatorname{Sim}\left(\mathrm{T}_{\mathrm{i}}, \mathrm{C}_{\mathrm{j}}\right)=\mathrm{w}_{1}+\mathrm{w}_{2}+\ldots+\mathrm{w}_{\mathrm{m}}$

\section{Cluster Construction:}

Informally, each transaction is evaluated in terms of the following criteria;

a) We sssign a new transaction $T_{i}$ to cluster $C_{j}$ which has the minimum $\operatorname{Sim}\left(\mathrm{T}_{\mathrm{i}}, \mathrm{C}_{\mathrm{j}}\right.$ ) value among open clusters (a cluster is open if has not exceeded its expected size in terms of number of transactions).

b) Each new allocation to a cluster $C_{j}$, updates the cluster centroid $C_{j}$. All already existing common items' weight is increased by 1 , and the other new items are added to $C_{j}$ with the weight of value 1 .

Reasoning about the size of clusters: based on the observation in section 4.1, the cluster sizes should be well balanced.

The pseudo incremental clustering algorithm is described as the following

Input: Transaction database: $\mathrm{D} ; \mathrm{k}$ - number of output clusters

Output: $k$ clusters based on the above criteria for Partition approach.

\section{Begin}

1. Assign the first $\mathrm{k}$ transactions to all $\mathrm{k}$ clusters, and initialize the all Cluster Centroids: $\left\{\mathrm{C}_{1}, \mathrm{C}_{2}, \ldots, \mathrm{C}_{\mathrm{k}}\right\}$

2. Consider the next $\mathrm{k}$ transactions: $\left\{\mathrm{T}_{1}, \mathrm{~T}_{2}, \ldots, \mathrm{T}_{\mathrm{k}}\right\}$. These $\mathrm{k}$ transactions are assigned to $\mathrm{k}$ different clusters. These operations are done based on the following criteria: (i) the minimum similarity between the new transaction and the suitable clusters; (ii) the sizes of these clusters are controlled to keep the balance. The following are more detail about this processing.

Let $C^{\text {run }}=\left\{C_{1}, C_{2}, \ldots, C_{k}\right\}$ is a set of all k clusters; $T^{\text {run }}=\left\{T_{1}, T_{2}, \ldots, T_{k}\right\}$

For each transaction $\mathrm{T}_{\mathrm{i}}$ in $\mathrm{T}^{\mathrm{run}}: \mathrm{T}_{1}$ to $\mathrm{T}_{\mathrm{k}}$ Begin

a) Calculate the similar functions between $T_{i}$ and all the clusters in $C^{\text {run }}$; determine the minimum similar function value, denoted $\operatorname{Sim}\left(T_{i}, C_{j}\right)$

b) Assign $T_{i}$ to cluster $C_{j}$ which has the minimum $\operatorname{Sim}\left(T_{i}, C_{j}\right)$ value. Update the cluster centroid $\mathrm{C}_{\mathrm{j}}$

c) Remove $C_{j}$ from the set of all the suitable clusters in order to keep the same size constraint. $C^{\text {run }}=C^{\text {run }}-\left\{C_{j}\right\}$;

End

3. Repeat step 2 till all transactions in D are clustered

\section{End}


The time complexity of this incremental clustering algorithm is about $\mathrm{O}(|\mathrm{D}| * \mathrm{k} * \mathrm{~m})$ where $|\mathrm{D}|$ is the number of all transactions, $\mathrm{k}$ is the given number of clusters, and $\mathrm{m}$ is the number of all items in D.

\section{Experiments}

In this section, we conducted experiments on: one synthetic data set [1], and 3 real data sets [13]. These data sets are converted to format as the above definitions.

Table 1. The characteristics of data sets

\begin{tabular}{|c|c|r|r|}
\hline Data sets & Transactions & Items & DB Size ( MB) \\
\hline T10I4D100K & $100 \mathrm{~K}$ & 870 & 4 \\
\hline WebView-1 & $26 \mathrm{~K}$ & 492 & 0.7 \\
\hline WebView-2 & $52 \mathrm{~K}$ & 3335 & 2 \\
\hline BMS-POS & $435 \mathrm{~K}$ & 1657 & 10 \\
\hline
\end{tabular}

Our goal is to compare the cardinality of the outputs from two phases of the Partitioning algorithm; at the Local level and the Global level, before and after application of our pre-processing. Firstly, data set is partitioned into fragments; secondly the Apriori algorithm (by Zhu T. [12]) is applied to find Local frequent itemsets $\left(\mathrm{LP}_{\mathrm{i}}\right.$ ) for each fragment. Subsequently, union of these LPi generates the Global candidates.

Resulting figures for each data set are represented in following template table 2 . The $2^{\text {nd }}, 3^{\text {rd }}$ and $4^{\text {th }}$ columns' names indicate three techniques for data preparation: Sequent fragments correspond to loading clusters with original data, Skipping fragments are constructed as described in section 5.1; and the Clustering fragments are the pre-processed data as presented by our clustering method described in section 5.2.

The data sets used are indicated on the top of each table segment. We present three different scenarios; each data set is partitioned into 1, 2 and 5 fragments. The Sequent column represents the numbers of the Local level $\left(\mathrm{LP}_{1}, \mathrm{LP}_{2}, \ldots, \mathrm{LP}_{\mathrm{n}}\right)$, the number of Global candidates. Note that this figure is presented by showing its two components; for example, $16+(378)$ indicates that there are 16 candidates to be checked and $\mathbf{3 7 8}$ common candidates don't need additional check.

Using the same convention, the Skipping and Clustering columns represent the figures for the Skipping technique and the Clustering pre-processing, respectively.

As can be seen from Table 2 and 3, there are big gains from the careful data preprocessing. Further, to discuss the impact of threshold level, let us denote the cardinality of checked Global candidate set as $\left|C_{n}{ }^{G}\right|$, where $n$ is the number of fragments. $\mid \mathrm{C}_{\mathrm{n}} \mathrm{G}^{\mathrm{G}}$ is reduced for all data sets for all support thresholds. For example, if T10I4D100k is partitioned into 2 fragments, $\left|\mathrm{C}_{2}{ }^{\mathrm{G}}\right|$ decreases from $\mathbf{1 6}$ for Sequent to 3 for Clustering with the support threshold $\mathbf{0 . 0 1}$. This reduction is also present when considering other real data sets that are partitioned into 2 fragments. Its value reduces from $\mathbf{1 , 8 2 0}$ to $\mathbf{3 4 8}$ with the threshold $\mathbf{0 . 0 0 5}$ for very large data set BMS-POS. Moreover, if data sets are partitioned into 5 fragments, this gap among 3 techniques is even greater. For example, if T10I4D100k is partitioned into 5 fragments, $\left|C_{5}{ }^{G}\right|$ decreases from 48 for Sequent to 24 for Clustering with the threshold $\mathbf{0 . 0 1}$, and 698 to 373 with 
Table 2. The figures with a threshold 0.01

\begin{tabular}{|c|c|c|c|}
\hline & Sequent & Skipping & Clustering \\
\hline \multicolumn{4}{|c|}{ T10I4D100K } \\
\hline \multicolumn{4}{|c|}{ 1-fragment: $\mathbf{3 8 5}$ Frequent Itemsets } \\
\hline \multicolumn{4}{|c|}{2 fragments } \\
\hline LP1 & 385 & 387 & 385 \\
\hline LP2 & 387 & 386 & 386 \\
\hline $\mathrm{C}_{2}^{\mathrm{G}}$ & $16+(378)$ & $17+(378)$ & $3+(384)$ \\
\hline \multicolumn{4}{|c|}{5 fragments } \\
\hline LP1 & 392 & 386 & 387 \\
\hline LP2 & 381 & 388 & 387 \\
\hline LP3 & 393 & 388 & 384 \\
\hline LP4 & 386 & 387 & 388 \\
\hline LP5 & 390 & 391 & 388 \\
\hline $\mathrm{C}_{5}^{\mathrm{G}}$ & $48+(366)$ & $57+(362)$ & $24+(375)$ \\
\hline \multicolumn{4}{|c|}{ WebView-1 } \\
\hline \multicolumn{4}{|c|}{ 1-fragment: 208 Frequent Itemsets } \\
\hline LP1 & 227 & 241 & 210 \\
\hline LP2 & 229 & 201 & 213 \\
\hline $\mathrm{C}_{2}^{\mathrm{G}}$ & $152+152)$ & $116+(163)$ & $17+(203)$ \\
\hline \multicolumn{4}{|c|}{5 fragments } \\
\hline LP1 & 284 & 250 & 226 \\
\hline LP2 & 197 & 230 & 221 \\
\hline LP3 & 241 & 254 & 213 \\
\hline LP4 & 255 & 242 & 207 \\
\hline LP5 & 266 & 205 & 205 \\
\hline $\mathrm{C}_{5}^{\mathrm{G}}$ & $425+(92)$ & $228+(141)$ & $74+(181)$ \\
\hline \multicolumn{4}{|c|}{ WebView-2 } \\
\hline \multicolumn{4}{|c|}{ 1-fragment: 186 Frequent Itemsets } \\
\hline LP1 & 279 & 156 & 192 \\
\hline LP2 & 221 & 236 & 179 \\
\hline $\mathrm{C}_{2}{ }^{\mathrm{G}}$ & $292+(104)$ & $120+(136)$ & $19+(176)$ \\
\hline \multicolumn{4}{|c|}{5 fragments } \\
\hline LP1 & 133 & 197 & 188 \\
\hline LP2 & 558 & 182 & 209 \\
\hline LP3 & 384 & 184 & 193 \\
\hline LP4 & 244 & 180 & 169 \\
\hline LP5 & 227 & 247 & 195 \\
\hline $\mathrm{C}_{5}{ }^{\mathrm{G}}$ & $756+(55)$ & $157+(135)$ & $64+(160)$ \\
\hline \multicolumn{4}{|c|}{ BMS-POS } \\
\hline \multicolumn{4}{|c|}{ 1-fragment: $\mathbf{1 , 5 0 3}$ Frequent Itemsets } \\
\hline LP1 & 1,400 & 1,353 & 1,512 \\
\hline LP2 & 1,662 & 1,680 & 1,498 \\
\hline $\mathrm{C}_{2}^{\mathrm{G}}$ & $\begin{array}{l}390+ \\
(1,336) \\
\end{array}$ & $\begin{array}{l}341+ \\
(1,346) \\
\end{array}$ & $\begin{array}{l}60+ \\
(1,475)\end{array}$ \\
\hline \multicolumn{4}{|c|}{5 fragments } \\
\hline LP1 & 1,996 & 1,719 & 1,150 \\
\hline LP2 & 1,334 & 1,146 & 1,471 \\
\hline LP3 & 744 & 1,639 & 1,864 \\
\hline LP4 & 1,348 & 1,810 & 1,822 \\
\hline LP5 & 2,885 & 1,377 & 1,364 \\
\hline $\mathrm{C}_{5}{ }^{\mathrm{G}}$ & $\begin{array}{l}2,263+ \\
(689)\end{array}$ & $\begin{array}{c}950+ \\
(1,067)\end{array}$ & $\begin{array}{c}894+ \\
(1,121) \\
\end{array}$ \\
\hline
\end{tabular}

Table 3. The figures with threshold 0.005

\begin{tabular}{|c|c|c|c|}
\hline & Sequent & Skipping & Clustering \\
\hline \multicolumn{4}{|c|}{ T10I4D100K } \\
\hline \multicolumn{4}{|c|}{ 1-fragment: 1,073 Frequent Itemsets } \\
\hline \multicolumn{4}{|c|}{2 fragments } \\
\hline LP1 & 1,079 & 1,101 & 1,068 \\
\hline LP2 & 1,101 & 1,077 & 1,092 \\
\hline $\mathrm{C}_{2}{ }^{\mathrm{G}}$ & $158+(1,011)$ & $148+(1,015)$ & $70+(1,045)$ \\
\hline \multicolumn{4}{|c|}{5 fragments } \\
\hline LP1 & 1,150 & 1,181 & 1,089 \\
\hline LP2 & 1,141 & 1,074 & 1,110 \\
\hline LP3 & 1,248 & 1,091 & 1,059 \\
\hline LP4 & 1,110 & 1,122 & 1,135 \\
\hline LP5 & 1,120 & 1,135 & 1,098 \\
\hline $\mathrm{C}_{5}{ }^{\mathrm{G}}$ & $698+(893)$ & $578+(889)$ & $373+(941)$ \\
\hline \multicolumn{4}{|c|}{ WebView-1 } \\
\hline \multicolumn{4}{|c|}{ 1-fragment: 633 Frequent Itemsets } \\
\hline LP1 & 644 & 774 & 659 \\
\hline LP2 & 755 & 612 & 641 \\
\hline $\mathrm{C}_{2}^{\mathrm{G}}$ & $503+(448)$ & $416+(485)$ & $94+(603)$ \\
\hline \multicolumn{4}{|c|}{5 fragments } \\
\hline LP1 & 1,107 & 771 & 779 \\
\hline LP2 & 489 & 842 & 733 \\
\hline LP3 & 839 & 941 & 676 \\
\hline LP4 & 894 & 769 & 663 \\
\hline LP5 & 977 & 517 & 597 \\
\hline $\mathrm{C}_{5}{ }^{\mathrm{G}}$ & $1,806+(271)$ & $1,069+(374)$ & $497+(493)$ \\
\hline \multicolumn{4}{|c|}{ WebView-2 } \\
\hline \multicolumn{4}{|c|}{ 1-fragment: 996 Frequent Itemsets } \\
\hline LP1 & 1,980 & 738 & 1,064 \\
\hline LP2 & 1,058 & 1,422 & 941 \\
\hline $\mathrm{C}_{2}{ }^{\mathrm{G}}$ & $2,150+(444)$ & $808+(676)$ & $191+(907)$ \\
\hline \multicolumn{4}{|c|}{5 fragments } \\
\hline LP1 & 682 & 1,130 & 1,067 \\
\hline LP2 & 8,546 & 997 & 1,355 \\
\hline LP3 & 2,899 & 911 & 986 \\
\hline LP4 & 1,271 & 957 & 791 \\
\hline LP5 & 1,257 & 1,412 & 1,069 \\
\hline $\mathrm{C}_{5}{ }^{\mathrm{G}}$ & $10,007+(230)$ & $1,114+(625)$ & $751+(723)$ \\
\hline \multicolumn{4}{|c|}{ BMS-POS } \\
\hline \multicolumn{4}{|c|}{ 1-fragment: 6,017 Frequent Itemsets } \\
\hline LP1 & 5,419 & 5,311 & 6,024 \\
\hline LP2 & 6,709 & 6,729 & 5,972 \\
\hline $\mathrm{C}_{2}^{\mathrm{G}}$ & $\begin{array}{l}1,820+ \\
(5,154)\end{array}$ & $\begin{array}{l}1,468+ \\
(5,286)\end{array}$ & $348+(5,824)$ \\
\hline \multicolumn{4}{|c|}{5 fragments } \\
\hline LP1 & 8,480 & 7,014 & 4,339 \\
\hline LP2 & 4,975 & 4,290 & 5,932 \\
\hline LP3 & 2,541 & 6,619 & 7,530 \\
\hline LP4 & 5,177 & 7,315 & 7,443 \\
\hline LP5 & 12,755 & 5,287 & 5,289 \\
\hline $\mathrm{C}_{5}^{\mathrm{G}}$ & $\begin{array}{l}10,718+ \\
(2,346)\end{array}$ & $\begin{array}{l}4,353+ \\
(3,956)\end{array}$ & $\begin{array}{l}4,075+ \\
(4,191)\end{array}$ \\
\hline
\end{tabular}


the threshold $\mathbf{0 . 0 0 5}$, respectively. Exceptional performance for WebView-2 data set with the threshold $\mathbf{0 . 0 0 5}$ the reduction is from $\mathbf{1 0 , 0 0 7}$ to only $\mathbf{7 5 1}$ when data set is partitioned into 5 fragments.

Hence naturally, another interesting and encouraging trend can be found in the growth of the number of common candidates between $\mathrm{LP}_{\mathrm{i}}$ for fragmented data sets. For example, if data sets are partitioned into 5 fragments, this common number increases from 689 to 1,121 for BMS-POS with the threshold $\mathbf{0 . 0 1}$ as well as from 230 to $\mathbf{7 2 3}$ for WebView-2 with the threshold $\mathbf{0 . 0 0 5}$.

In summary, the figures from 2 above tables show that the Clustering preprocessing technique can significantly improve the Partitioning approach. It is delivered in form of two strongly related benefits; reduction of the number of Global candidates requiring the final check and increase of the common candidates numbers that don't require any additional checks.

\section{Conclusion}

This paper considers a new approach for further performance improvements in frequent itemsets computation. Based on the original Partition algorithm, we show that the composition of fragments and the number of fragments generated, impact on the size of the data used by this algorithm.

We propose a pre-processing method (an incremental clustering algorithm), mainly to demonstrate that there is potential in the direction of performance improvement. Figures from the experiments show that this pre-processing offers good benefits already. The main question which still deserves consideration is related to the identification of methods that will deliver an even better partition for the original data sets.

Acknowledgment. We wish to thank the Data Mining group at ITEE School - The University of Queensland and the anonymous reviewers for suggestions.

\section{References}

[1] Agrawal R., Srikant R.: Fast algorithms for mining association rules. Proc. $20^{\text {th }}$ Int. Conf. Very Large Data Bases, Morgan Kaufmann, 1994 (487 - 499)

[2] Savasere A., Omiecinski E., Navathe S.: An efficient algorithms for mining association rules in large database. Proc. $21^{\text {th }}$ Int. Conf. Very Large Data Bases, Swizerland, 1995

[3] Goethals B.: Survey on frequent pattern mining. University of Helsinki, 2002

[4] Lin J.L., Dunham M.H.: Mining association rules: Anti-skew algorithms. Proc. $14^{\text {th }}$ IEEE Int. Conf. on Data Engineering, Florida, 1998

[5] Agrawal R., Imielinski T., Swami A.N.: Mining association rules between sets of items in lagre database. Proc. 1993 ACM SIGMOD Int. Conf. on Management of Data, 1993

[6] Brin S., Motwani R., Ullman D.J., Tsur S.: Dynamic Itemset Counting and implication rules for masket basket data. Proc. ACM SIGMOD 1997 Int. Conf. on Management of Data, 1997 (255 - 264)

[7] Zaki M.J.: Scalable algorithms for association mining. IEEE Transactions on Knowledge and Data Engineering, 12(3): 372-390, 2000 
[8] Han J., Pei J., Yin Y., Mao R.: Mining frequent patterns without candidate generation: A frequent-pattern tree approach. Data Mining and Knowledge Discovery, Kluwer Academic Publishers, (8): 53-87, 2004

[9] Mueller A.: Fast sequential and parallel algorithm for association rules mining: A comparison. Technical Report CS-TR-3515, University of Maryland, 1995

[10] Son N. Nguyen: Data partitioning approach into selected data mining problems. $\mathrm{PhD}$ Confirmation report, The University of Queensland, Australia, 2005

[11] Pudi V., Haritsa J.: ARMOR: Association rule mining based on Oracle. Workshop on Frequent Itemset Mining Implementations (FIMI'03 in conjunction with ICDM'03), 2003

[12] Zhu T.: The Apriori algorithm implementation, http://www.cs.ualberta.ca/ tszhu/

[13] Ron Kohavi, Carla Brodley, Brian Frasca, Llew Mason, and Zijian Zheng. KDD-Cup 2000 organizers' report: Peeling the onion. SIGKDD Explorations, 2(2):86-98, 2000 